\title{
Impact of organic overlayers on a-Si:H/c-Si surface potential
}

Johannes P. Seif, Bjoern Niesen, Andrea Tomasi, Christophe Ballif, and Stefaan De Wolf

Citation: Appl. Phys. Lett. 110, 151601 (2017); doi: 10.1063/1.4980047

View online: http://dx.doi.org/10.1063/1.4980047

View Table of Contents: http://aip.scitation.org/toc/apl/110/15

Published by the American Institute of Physics

\section{Articles you may be interested in}

Optically read displacement detection using phase-modulated diffraction gratings with reduced zeroth-order reflections

Appl. Phys. Lett. 110, 151104151104 (2017); 10.1063/1.4979541

Carbon nanotube stabilized single layer graphene cantilevers

Appl. Phys. Lett. 110, 151901151901 (2017); 10.1063/1.4979837

BiFeO3-doped (K0.5,Na0.5)(Mn0.005,Nb0.995)O3 ferroelectric thin film capacitors for high energy density storage applications

Appl. Phys. Lett. 110, 152901152901 (2017); 10.1063/1.4980113

Silicon surface passivation by polystyrenesulfonate thin films

Appl. Phys. Lett. 110, 083904083904 (2017); 10.1063/1.4976949

Difference of near-interface strain in $\mathrm{SiO} 2$ between thermal oxides grown on $4 \mathrm{H}-\mathrm{SiC}$ by dry-O2 oxidation and $\mathrm{H} 2 \mathrm{O}$ oxidation characterized by infrared spectroscopy

Appl. Phys. Lett. 110, 152104152104 (2017); 10.1063/1.4980093

Coherent radiation at the fundamental frequency by a Smith-Purcell free-electron laser with dielectric substrate Appl. Phys. Lett. 110, 151108151108 (2017); 10.1063/1.4980046
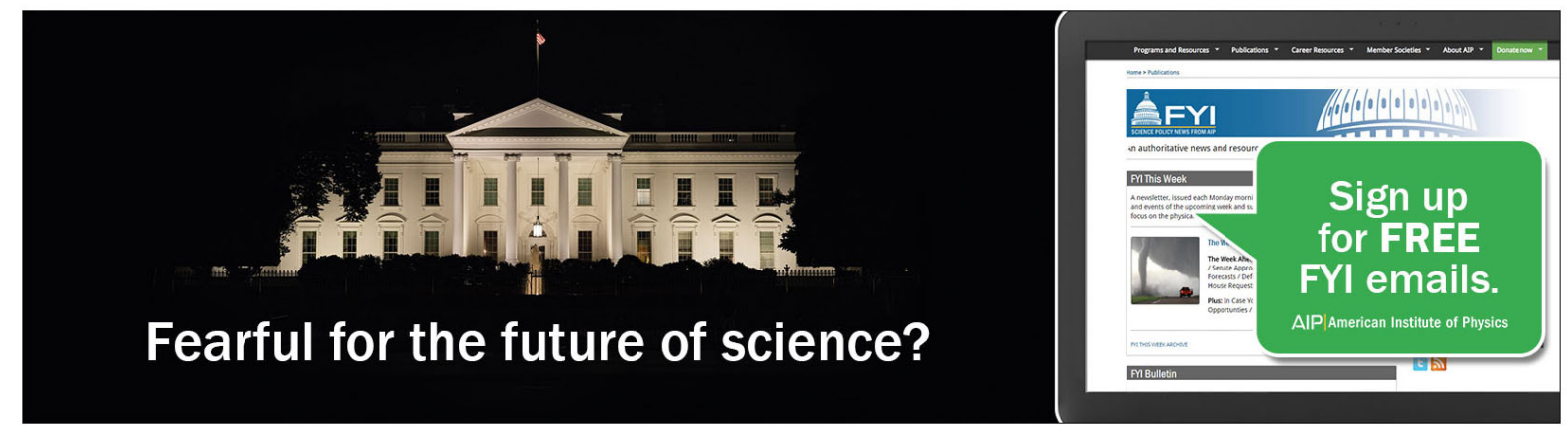


\title{
Impact of organic overlayers on a-Si:H/c-Si surface potential
}

\author{
Johannes P. Seif, ${ }^{1}$ Bjoern Niesen, ${ }^{1}$ Andrea Tomasi, ${ }^{1}$ Christophe Ballif, ${ }^{1}$ \\ and Stefaan De Wolf ${ }^{2}$ \\ ${ }^{1}$ Ecole Polytechnique Fédérale de Lausanne (EPFL), Institute of Microengineering (IMT), Photovoltaics \\ and Thin-Film Electronics Laboratory (PV-Lab), Neuchâtel 2002, Switzerland \\ ${ }^{2}$ King Abdullah University of Science and Technology (KAUST), KAUST Solar Center (KSC), \\ Thuwal 23955-6900, Saudi Arabia
}

(Received 2 December 2016; accepted 30 March 2017; published online 11 April 2017)

\begin{abstract}
Bilayers of intrinsic and doped hydrogenated amorphous silicon, deposited on crystalline silicon $(c-\mathrm{Si})$ surfaces, simultaneously provide contact passivation and carrier collection in silicon heterojunction solar cells. Recently, we have shown that the presence of overlaying transparent conductive oxides can significantly affect the $c$-Si surface potential induced by these amorphous silicon stacks. Specifically, deposition on the hole-collecting bilayers can result in an undesired weakening of contact passivation, thereby lowering the achievable fill factor in a finished device. We test here a variety of organic semiconductors of different doping levels, overlaying hydrogenated amorphous silicon layers and silicon-based hole collectors, to mitigate this effect. We find that these materials enhance the $c$-Si surface potential, leading to increased implied fill factors. This opens opportunities for improved device performance. Published by AIP Publishing.

[http://dx.doi.org/10.1063/1.4980047]
\end{abstract}

Silicon heterojunction (SHJ) solar cells feature remarkably high operating voltages thanks to the excellent crystalline silicon $(c-\mathrm{Si})$ surface passivation provided by their electron and hole contacts. Such high-performance passivation is primarily enabled by the use of thin intrinsic hydrogenated amorphous silicon $(a-\mathrm{Si}: \mathrm{H})$ layers deposited on the wafer surfaces, providing chemical surface passivation. Photogenerated electrons and holes are then selectively collected through the $a-\mathrm{Si}: \mathrm{H} / c-\mathrm{Si}$ interfaces thanks to the presence of $n$-type or $p$-type $a$-Si:H overlayers, respectively, inducing the necessary $c$-Si surface potential to do so; the surface potential is defined as the energy difference obtained comparing the lowest electronic states in the bulk of the semiconductor (here $c$-Si) and its surface. The surface potential affects the carrier statistics close to the c-Si surface and consequently also the field effect passivation. These welldesigned, intrinsic/doped $a$-Si:H bilayers collect only one type of carrier, while repelling the opposite type. Therefore, these stacks yield simultaneously efficient carrier collection and improved surface passivation, thanks to the induced field-effects complementing the usually already outstanding chemical surface passivation given by the thin intrinsic $a$ $\mathrm{Si}: \mathrm{H}$ layers. In finished devices, these $a-\mathrm{Si}: \mathrm{H}$ bilayers are completed into electrical contacts by capping with transparent electrodes, fulfilling at least two functions: they warrant unhindered carrier transport to the metallic electrodes, needed for current extraction, ${ }^{1}$ and maximize light coupling into the silicon wafer, acting as an antireflection layer at the front of the device or dielectric spacer at the rear. ${ }^{2}$ The most commonly used transparent electrodes for high-efficiency devices are transparent conductive oxides (TCOs).

At high excess-carrier densities $(\Delta n)$, high-efficiency solar cells are usually only limited by Auger and radiative recombination. However, these intrinsic recombination pathways play a lesser role at the moderate $\Delta n$ values, those of the actual working point of the device (i.e., between $10^{14} \mathrm{~cm}^{-3}$ and $10^{15} \mathrm{~cm}^{-3}$ ). This is mainly due to the yet imperfect chemical passivation of the employed contacting stacks. This is one of the main reasons why the actual fill factor $(F F)$ of a device is often significantly lower than its maximum theoretical $F F$ values of about $89 \%$ (assuming a silicon wafer with a resistivity of $1 \Omega \mathrm{cm}$, a thickness of $100 \mu \mathrm{m}$, and a photogenerated current of $\left.37 \mathrm{~mA} / \mathrm{cm}^{2}\right) .{ }^{3-5}$ Whereas the equilibrium defect density of the thin intrinsic $a-\mathrm{Si}: \mathrm{H}$ buffer layers may set an upper limit to the maximal achievable chemical surface passivation with such layers, further improvements in passivation can be achieved by enhancing the field-effect induced by the intrinsic/doped $a$-Si:H film bilayers. Practically, this implies the improvement of the $c$-Si surface potential, inducing the properties of the doped overlayers, ${ }^{6}$ which advocates for keeping them sufficiently thick. ${ }^{7}$ However, from the optical perspective, such stacks should be kept as thin as possible, at least when located at the front side of the device. This tradeoff explains why the doped/intrinsic $a$-Si:H bilayers are usually kept very thin $(10-20 \mathrm{~nm}$ for the full stack) in highperformance devices. ${ }^{8-10}$ This, combined with the specific band energetics of the applied TCOs, can result in an altered $c$-Si surface potential, thus the field-effect, however, modifying the carrier recombination statistics at the interface. ${ }^{11,12}$ This will be explained in more detail later in this paper, on the basis of the results of the organic overlayers studied here.

Commonly used TCOs in SHJ solar cells are based on either indium oxide or zinc oxide. ${ }^{13}$ Irrespective of the specific material choice, all these materials should be gently deposited at sufficiently low temperatures, causing no damage to underlying films and interfaces. ${ }^{11,14}$ Most of the commonly used TCOs are of $n$-type. Also, $p$-type materials exist, albeit so far usually with a much poorer transparency/conductivity trade-off, compared to their $n$-type counterparts. ${ }^{13,15}$ However, despite being $n$-type, the "standard" TCOs usually exhibit relatively high work function (WF) values, ${ }^{16}$ which has important consequences for interface 
engineering. For example, the TCO WF values are typically higher than the WF values of $n$-type $c$-Si substrates but lower than the WF values of $p$-type $a$-Si:H films. This implies that-in theory-TCOs will collect holes when directly deposited on $c$-Si, yet not very efficiently. This explains why $p$-type $a$-Si:H films are usually inserted to increase the holecollecting efficiency. However, this can, in turn, result in band alignment issues, in terms of inefficient intra-band tunneling at the interface between the TCO and the $p$-type $a$-Si:H film ${ }^{17}$ and in terms of surface passivation losses. The latter is manifested by a reduced $c$-Si surface potential (and thus field effect), resulting in a reduced effective minoritycarrier lifetime $\left(\tau_{\text {eff }}\right)$ at low-to-moderate $\Delta n$ values and hence potentially lower fill factors $(F F) .^{7,11,12,18-22}$ This issue may be circumvented by employing an additional buffer layer that features higher WF values, compared to the $p$-type $a$-Si:H hole collectors. In this context, hole-collecting organic semiconductors (OSCs) are promising candidates to be applied at the hole collecting side in order to improve the band alignment at the wafer interface. In the past, OSCs have already been applied in optoelectronic devices such as organic light-emitting diodes and solar cells. ${ }^{23-29}$ Yet, they were mainly used for thin-film photovoltaic devices of moderate conversion efficiency. Only recently, they started to find their way also into $c$-Si-related applications. ${ }^{30-32}$ Specifically, Schmidt ${ }^{30}$ and Zielke et al. ${ }^{31}$ showed the applicability of OSCs in silicon-based cells by using poly(3,4-ethylenedioxythiophene):polystyrene sulfonate (PEDOT:PSS), but so far, the effect of such a layer on surface passivation remains unknown.

In our study, we investigate the effects of poly(N-vinylcarbazole) (PVK) on silicon surface passivation quality. PVK is a $p$-type semiconducting polymer commonly used as hole-transport or a host material in organic light-emitting diodes. Its highest occupied (HOMO) and lowest unoccupied molecular orbitals (LUMO) are at $-5.8 \mathrm{eV}$ and $-2.2 \mathrm{eV}$, respectively. ${ }^{33}$ It has been shown that the hole conductivity in PVK can effectively be enhanced by $p$-type dopants. ${ }^{34}$ Here, we blend PVK with lithium salt, which has been reported to $p$-type dope a wide range of polymeric and small-molecular organic semiconductors. ${ }^{35,36}$

For our study, we concentrate on symmetrically passivated $c$-Si wafers and pay specific attention to the evolution of $\tau_{\text {eff }}$ upon PVK deposition. We focus our investigations on double-side-polished (DSP) $n$-type [float-zone, (111), 255-305 $\mu \mathrm{m}, 1-5 \Omega \mathrm{cm}] c$-Si wafers. These samples received device-relevant symmetric passivation $[a-\mathrm{Si}: \mathrm{H}(i) /$ $a$-Si:H(i), hereafter referred to as $i / i$, thickness $\sim 8 \mathrm{~nm}$ on the DSP, measured by spectroscopic ellipsometry] or bilayers $[a-\mathrm{Si}: \mathrm{H}(i n) / a-\mathrm{Si}: \mathrm{H}($ in $)$, in/in, thickness $\sim 15 \mathrm{~nm}$ or $a$-Si:H(ip)/a-Si:H(ip), ip/ip, thickness $\sim 12 \mathrm{~nm}$ ] deposited in a medium-area plasma enhanced chemical vapor deposition (PECVD) reactor. More details are reported elsewhere. ${ }^{37,38}$ For the sake of simplicity, we refer to the samples in shorthand notation, e.g., an $n$-type wafer passivated with symmetric ip/ip bilayers and a PVK layer deposited one side is labeled as "ip-sample" (same for in- or $i$-samples). The structures investigated here are depicted in Fig. 1. Note that in order to investigate the effects of the PVK on the fieldeffect on either of the SHJ contacts, we processed

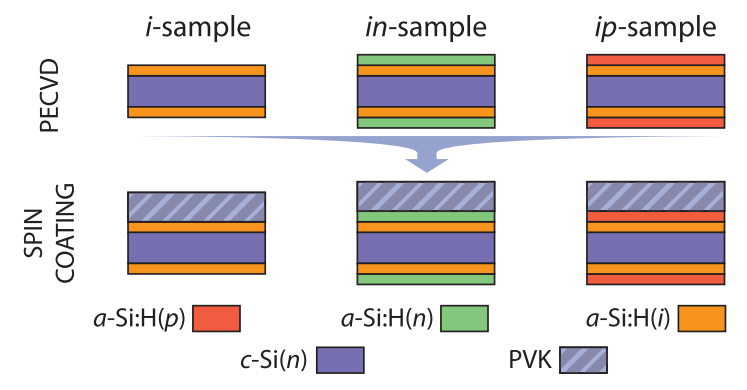

FIG. 1. Samples fabricated and measured in this study.

also in-samples, mainly for experimental crosschecking purposes. The ultimate goal, however, is the application of PVK on the hole-collecting side of our devices.

Following the PECVD depositions, PVK (dissolved in chlorobenzene at a concentration of $10 \mathrm{mg} / \mathrm{ml}$ ) doped with three different concentration levels of lithium bistrifluoromethanesulfonimidate was spin-cast on one side only (see Table I for details). The Li dopant concentration of the lowly doped film corresponds to $\sim 10 \mathrm{wt}$. $\%$, similar to the concentration typically used for many doped organic semiconductors, e.g., F4TCNQ-doped PVK ${ }^{34}$ or Li-doped Spiro-OMeTAD. ${ }^{35}$ For both these materials, a conductivity enhancement of about three orders of magnitude was observed at this doping level. For the highly doped layer, the doping concentration corresponds to 89 wt. \%. Each time, $150-200 \mu \mathrm{l}$ of the respective PVK solution was dispensed on the wafer and spun at $1000 \mathrm{rpm}$ for $5 \mathrm{~s}$, followed by $2000 \mathrm{rpm}$ for $60 \mathrm{~s}$. After this, the samples were annealed on a hotplate at $100^{\circ} \mathrm{C}$ for $\sim 5 \mathrm{~min}$ to evaporate the solvents. Both spin-casting and annealing were performed in a glove box in a controlled nitrogen atmosphere. We note that the highly doped PVK layer showed pinholes and contained large clusters and was not stable to thickness measurements by profilometry, jeopardizing its use for device applications. To assess the variations in field-effect passivation, standard lifetime measurements were performed with a photoconductance decay system before and after the spincoating step, taking care to center the samples the same way on the measurement tool for each measurement. ${ }^{3}$ Metallic contacts were not deposited in this study. Yet, the films are considered to be stable to withstand their application by thermal evaporation, a method commonly used to deposit metallic contacts on organic semiconductors.

In the following, we present the $\tau_{\text {eff }}$ results obtained on the samples described above. To start, we consider the simplest structure, namely, an $i$-sample. In this case, the deposition of a lowly doped PVK layer on one side of the wafer reduces $\tau_{\text {eff }}$ by $1 \mathrm{~ms}$ at a minority-carrier density of $10^{15}$ $\mathrm{cm}^{-3}$ as can clearly be seen in Fig. 2. The observed drop in

TABLE I. Details on PVK layers used in this study.

\begin{tabular}{lcc}
\hline \hline Doping & Dopant concentration $(\mu \mathrm{l} / \mathrm{ml})$ & Thickness $(\mathrm{nm})$ \\
\hline Undoped & 0 & $\sim 54$ \\
Lowly doped & 1.8 & $\sim 51$ \\
Highly doped & 17.5 & $\ldots{ }^{\mathrm{a}}$ \\
\hline \hline
\end{tabular}

${ }^{\mathrm{a}}$ Thickness not measurable by profilometry due to the unstable film. 


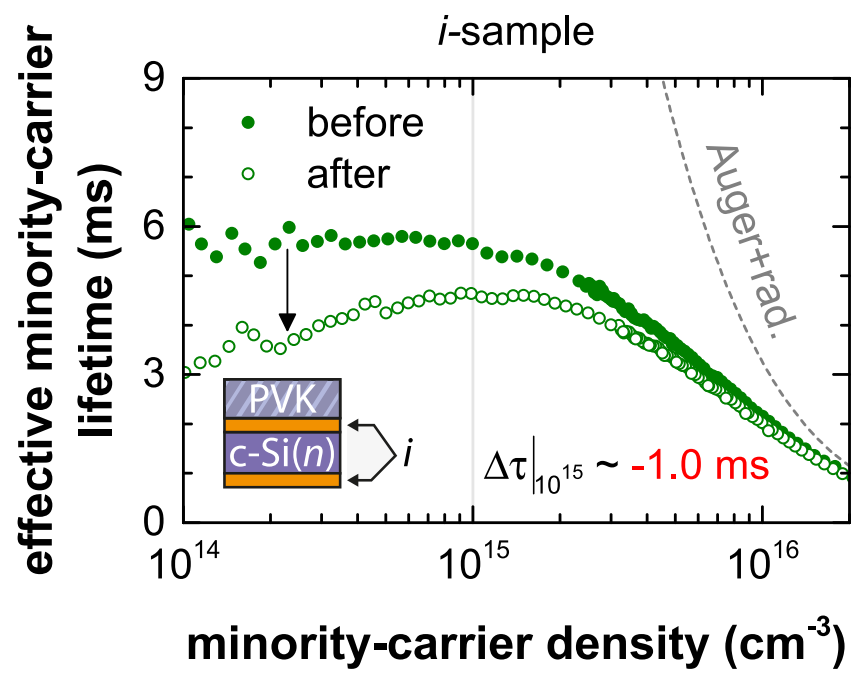

FIG. 2. Effective minority-carrier lifetime measured for a symmetrically $i / i$ passivated $n$-type wafer before (filled) and after (open) the deposition of lowly doped PVK layers applied to one side only. The dashed line represents the intrinsic recombination limit (Auger and radiative recombination). ${ }^{4}$ The inset shows the structure.

lifetime also reduces the implied $F F(i F F)$ calculated from these curves from $84.62 \%$ to $84.22 \%$. We remark that an identical passivation loss is observed when depositing an $a$-Si:H(p) layer on the $i$-layer. ${ }^{7,39}$

Going one step further and applying intrinsic and also doped amorphous silicon layers to the wafers, we obtain the results shown in Fig. 3. The given data again represent symmetrically passivated $n$-type wafers, before and after the deposition of the lowly doped PVK layers on one wafer side. Notably, depending on whether they exhibit in or ip a-Si:H layer bilayers, differences can be observed. While the data for the in-sample [Fig. 3(a)] show a small drop in $\tau_{\text {eff }}(-0.5 \mathrm{~ms}$ at $10^{15} \mathrm{~cm}^{-3}$ ), the opposite is true for the ip-sample, which shows an increase $\left[+0.9 \mathrm{~ms}\right.$ at $10^{15} \mathrm{~cm}^{-3}$, Fig. 3(b)]. For the in-sample, similar effects have been observed in the past using standard TCO layers, deposited very gently by atomic layer deposition. ${ }^{11}$

In order to understand the situation at hand, the band alignment situations before and after the deposition of the PVK are sketched for the in- and ip-samples in Fig. 4. The

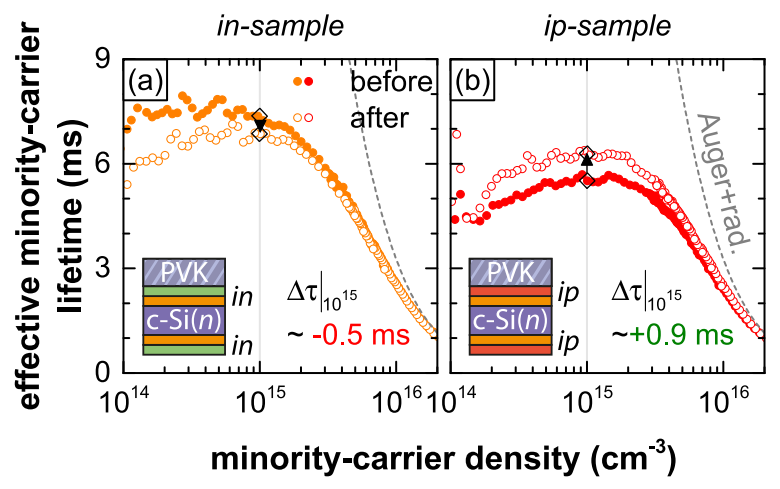

FIG. 3. Effective minority-carrier lifetimes measured for symmetrically passivated $n$-type wafers: (a) in- and (b) ip-samples before (filled) and after (open) the deposition of lowly doped PVK layers applied to one side only. The dashed line represents the intrinsic recombination limit (Auger and radiative recombination). ${ }^{4}$ The insets show the structure.
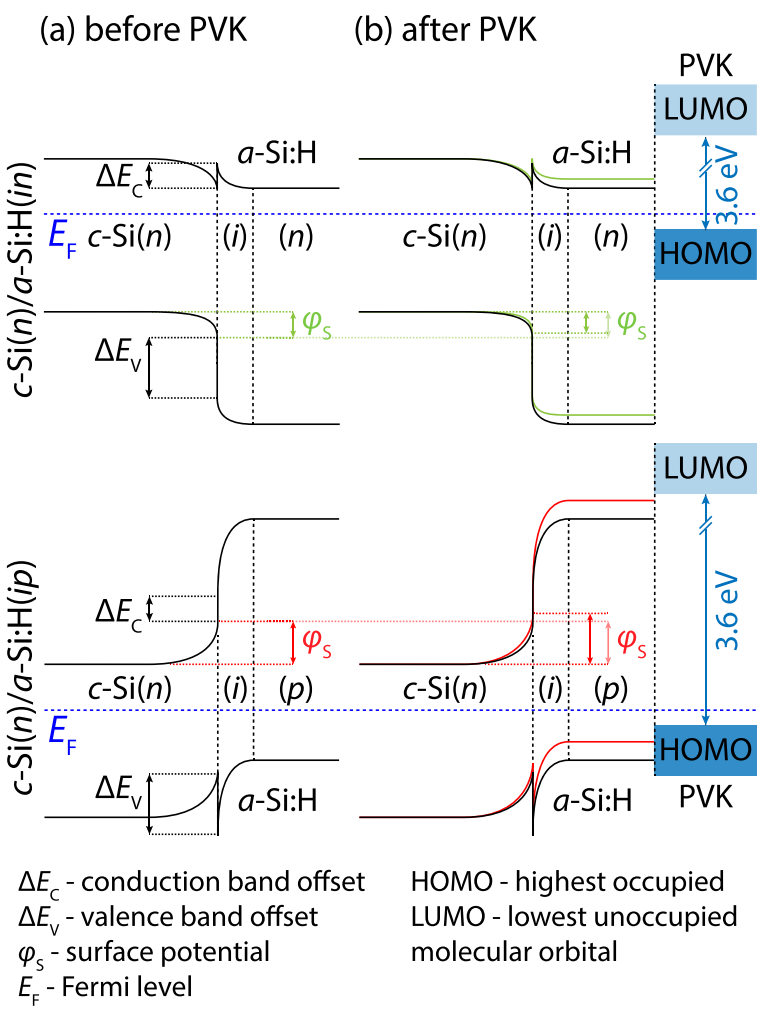

FIG. 4. Sketch of the band alignment of $n$-type crystalline silicon $[c-\mathrm{Si}(n)]$, passivated by amorphous silicon ( $a$-Si:H) either in the ip- or in-configuration (a) before and (b) after the deposition of the organic semiconductor, PVK, overlayer.

intrinsic layer alone (case not depicted here but similar to the in-case) provides excellent chemical surface passivation yet only a weak field effect. So, while the surface potential decreases for the $i$ - and the $i n$-case, the field effect passivation is reduced, and the opposite is true for the ip-case for which the surface potential is enhanced, and hence, the field effect passivation improves. As we do not assume any damage introduced by the deposition of the PVK, we ascribe the changes in lifetime to this modification of the $c$-Si surface potential and the resulting changes in field-effect passivation.

We focus now on the important upward trend in $\tau_{\text {eff }}$ for the $i p$-sample and the results obtained when coating them with differently doped PVK layers. For this, we refer to Table II and Fig. 5, respectively. While we already see an increase in $\tau_{\text {eff }}$ for the undoped PVK layer, this gain increases with doping in particular at low $\Delta n$ values. Again, the $p$-type OSCs

TABLE II. Implied $F F$ data obtained for all the ip- and in-samples processed in this study, before and after the deposition of the differently doped PVK layers.

\begin{tabular}{|c|c|c|c|c|c|}
\hline \multirow[b]{2}{*}{ PVK } & & \multicolumn{2}{|c|}{$i F F(\%)$} & \multicolumn{2}{|c|}{$i V_{\mathrm{oc}}(\mathrm{mV})$} \\
\hline & & in & ip & in & $i p$ \\
\hline \multirow[t]{2}{*}{ Undoped } & Before & 84.41 & 83.69 & 730 & 726 \\
\hline & After & 84.51 & 83.91 & 731 & 728 \\
\hline \multirow[t]{2}{*}{ Lowly doped } & Before & 84.78 & 84.02 & 731 & 729 \\
\hline & After & 84.53 & 84.45 & 731 & 730 \\
\hline \multirow[t]{2}{*}{ Highly doped } & Before & 84.72 & 84.19 & 730 & 731 \\
\hline & After & 84.47 & 84.85 & 730 & 731 \\
\hline
\end{tabular}




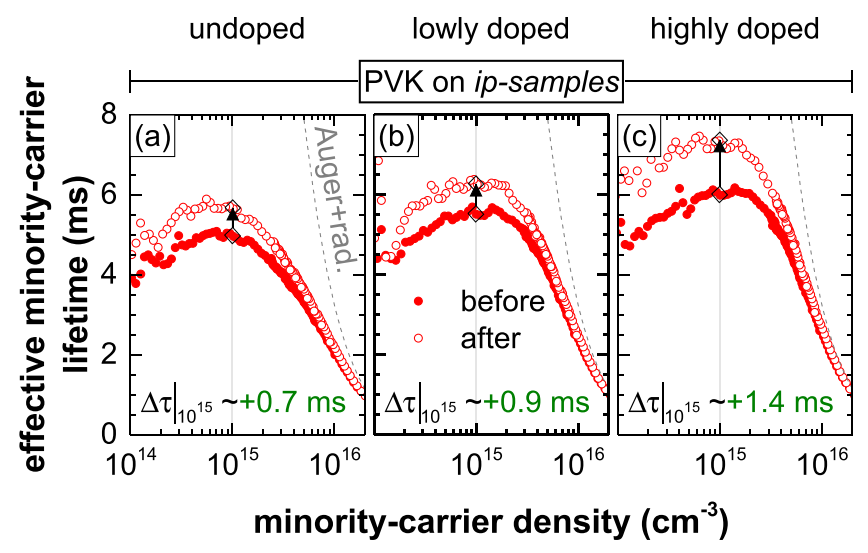

FIG. 5. Effective minority-carrier lifetimes measured for symmetrically ippassivated $n$-type wafers exhibiting differently doped PVK layers: (a) undoped, (b) lowly doped, and (c) highly doped. The filled symbols and open symbols represent the data before and after the deposition of the PVK. The dashed line represents the intrinsic recombination limit (Auger and radiative recombination). ${ }^{4}$

are expected to have this effect on the hole-collecting contact. Increasingly high doping levels in these $p$-type materials result in higher WFs that are indeed less detrimental for the field-effect passivation associated with $p$-type $a$-Si:H hole collectors. This pairs well with the contrasting observation of greater $\tau_{\text {eff }}$ losses at low $\Delta n$ values, for increasingly high doping levels in $n$-type TCOs. ${ }^{7}$ Furthermore, while the $i F F$ results obtained for the in-samples show a clear decrease by approximately $-0.3 \%$ absolute for the highly doped PVK, the ipsamples show a distinct increase- $+0.22 \%$ absolute for the undoped, $+0.43 \%$ for the lowly doped, and $+0.66 \%$ for the highly doped PVK - as expected from the $\tau_{\text {eff }}$ results. Applied in a device, this could enable better hole-collecting contacts and improved $F F$ values. It is important to note that these changes in $i F F$ have been obtained, while the implied opencircuit voltage $\left(i V_{\mathrm{oc}}\right)$ values remained virtually unchanged for both in- and ip-samples cases. For all samples processed here, the $i V_{\mathrm{oc}}$ values were in the range of $730 \mathrm{mV}$ with a variation of 1-2 $\mathrm{mV}$ before and after the deposition of the PVK. These small variations can be explained by the fact that even though we took specific care to place the samples always in the spot on the lifetime tester, they might not have been in the exact same position for each of the two measurements (before and after PVK).

A crucial ingredient for high-efficiency is the high passivation throughout the entire $\Delta n$ range, i.e., from the $\Delta n$ value associated with the working point of the device to the value attained in open-circuit conditions. ${ }^{22}$ Achieving this ideally leads to both high $F F$ values and high open-circuit voltages. Practically, for typical silicon heterojunction cells, this is not the case yet as they still suffer from the presence of interface states, insufficiently screened by the induced $c$ Si surface potential. ${ }^{7}$ The strategy presented here is to increase $c$-Si surface potential by inserting higher WF materials as buffer layers between the $a-\mathrm{Si}: \mathrm{H}$ and the TCO layers. These layers may mitigate the detrimental effects on the $c$-Si surface potential, induced by the TCO. In principle, OSCs could be used to replace the TCO layer, as it has been shown, for example, for organic solar cells using the highly doped polymer PEDOT:PSS, e.g., by Kim et al. ${ }^{40}$ If, however, OSC-TCO bilayers should be required, sufficiently thick
OSC layers are expected to efficiently screen the $c$-Si surface from the detrimental influence of the TCO. This is thanks to their lower relative permittivity and hence longer dielectric screening length (Debye length).

One promising candidate for more optimized WFs was presented here, namely, a $p$-type OSC material, improving the passivation in the low-to-moderate $\Delta n$ range. Earlier, Schmidt $^{30}$ and Zielke et al. $^{31}$ applied PEDOT:PSS (a holecollecting polymer) either at the front or rear of the $c$-Sibased device both as a hole collector and a transparent electrode. Despite the promising first results, significant parasitic absorption losses within the PEDOT:PSS layers offset their electrical potential, making them suitable for the application at the rear only. ${ }^{31}$ Polymers themselves may be highly transparent; however, as strong doping is required to increase the conductivity, absorption due to charge-transfer states in the visible and significant free-carrier absorption in the infrared may occur. ${ }^{34}$ For device architectures using a full-area metallic rear electrode polymer may, however, be beneficial. A full-area rear electrode relaxes the doping constraints, as only transversal conductance through the layer is important. Hence, a moderately doped layer may be used, alleviating potential free carrier absorption losses. Additionally, thanks to the low refractive index-inherent to most of these OSCs - their integration at the rear of silicon solar cells could even bring along optical benefits, as they might prevent plasmon-polariton-induced losses in the rear metal electrode. ${ }^{41-43}$ Finally, OSC films are typically deposited by a simple and soft technique, namely, solutioncoating and thermal evaporation, that preserves pristine $a$ $\mathrm{Si}: \mathrm{H}$ layer passivation, which is not always observed when using TCO deposition techniques such as sputtering. ${ }^{14}$

To summarize, despite their unfavorable effect on the passivation in $a$-Si:H(i)-passivated structures, we were able to show the potential of organic $p$-type polymers as essential components of hole-collecting contacts in $n$-type SHJ solar cells with improved contact passivation. In combination with intrinsic and $p$-doped $a$-Si:H layers, these organic materials yield very high $i F F$ values close to $85 \%$. Yet, future investigations will have to show whether the materials discussed here live up to their expectations and also whether the hole contact can be improved at a device level.

The authors gratefully acknowledge the technical support of Cédric Bucher and Lionel Domon. This work was funded by the EuroTech Universities Alliance in the framework of the Interface Science for Photovoltaics initiative, by Axpo Naturstrom Fonds, by the European Commission (FP7 project HERCULES, Contract No. 608498; FP7 project CHEETAH, Contract No. 609788), by the Office fédéral de l'énergie (OFEN), and by the Fonds National Suisse (FNS) Reequip program (Grant Nos. 206021_139135 and 206021_133832). The research reported in this publication was supported by funding the from King Abdullah University of Science and Technology (KAUST).

${ }^{1}$ S. De Wolf, A. Descoeudres, Z. C. Holman, and C. Ballif, Green 2(1), 7 (2012).

${ }^{2}$ Z. C. Holman, M. Filipič, A. Descoeudres, S. De Wolf, F. Smole, M. Topič, and C. Ballif, J. Appl. Phys. 113(1), 013107 (2013). 
${ }^{3}$ R. A. Sinton and A. Cuevas, Appl. Phys. Lett. 69(17), 2510 (1996).

${ }^{4}$ A. Richter, S. W. Glunz, F. Werner, J. Schmidt, and A. Cuevas, Phys. Rev. B 86(16), 1652021 (2012).

${ }^{5}$ A. Richter, M. Hermle, and S. W. Glunz, IEEE J. Photovoltaics 3(4), 1184 (2013).

${ }^{6}$ J. P. Seif, A. Descoeudres, G. Nogay, S. Hänni, S. Martin de Nicolas, N. Holm, J. Geissbühler, A. Hessler-Wyser, M. Duchamp, R. E. DuninBorkowski, M. Ledinsky, S. De Wolf, and C. Ballif, IEEE J. Photovoltaics 6, 1132 (2016).

${ }^{7}$ A. Tomasi, F. Sahli, J. P. Seif, L. Fanni, S. Martin de Nicolas, J. Geissbühler, B. Paviet-Salomon, S. Nicolay, L. Barraud, B. Niesen, S. De Wolf, and C. Ballif, IEEE J. Photovoltaics 6, 17-27 (2015).

${ }^{8}$ H. Fujiwara and M. Kondo, J. Appl. Phys. 101(5), 054516 (2007).

${ }^{9}$ M. Taguchi, E. Maruyama, and M. Tanaka, Jpn. J. Appl. Phys., Part 1 47(2), 814 (2008).

${ }^{10}$ Z. C. Holman, A. Descoeudres, L. Barraud, F. Zicarelli Fernandez, J. P. Seif, S. De Wolf, and C. Ballif, IEEE J. Photovoltaics 2(1), 7 (2012).

${ }^{11}$ B. Demaurex, J. P. Seif, S. Smit, B. Macco, W. M. M. E. Kessels, J. Geissbühler, S. De Wolf, and C. Ballif, IEEE J. Photovoltaics 4(6), 1387 (2014).

${ }^{12}$ R. Rößler, C. Leendertz, L. Korte, N. Mingirulli, and B. Rech, J. Appl. Phys. 113(14), 144513 (2013).

${ }^{13}$ M. Morales-Masis, S. De Wolf, R. Woods-Robinson, J. W. Ager, and C. Ballif, "Transparent Electrodes for Efficient Optoelectronics," Adv. Energy Mater. (published online).

${ }^{14}$ B. Demaurex, S. De Wolf, A. Descoeudres, Z. C. Holman, and C. Ballif, Appl. Phys. Lett. 101(17), 171604 (2012).

${ }^{15}$ X. Xu, J. Bullock, L. T. Schelhas, E. Z. Stutz, J. J. Fonseca, M. Hettick, V. L. Pool, K. F. Tai, M. F. Toney, X. Fang, A. Javey, L. H. Wong, and J. W. Ager, Nano Lett. 16(3), 1925 (2016).

${ }^{16}$ A. Klein, C. Körber, A. Wachau, F. Säuberlich, Y. Gassenbauer, R. Schafranek, S. P. Harvey, and T. O. Mason, Thin Solid Films 518(4), 1197 (2009).

${ }^{17}$ A. Kanevce and W. K. Metzger, J. Appl. Phys. 105(9), 094507 (2009).

${ }^{18}$ E. Centurioni and D. Iencinella, IEEE Electron Device Lett. 24(3), 177 (2003).

${ }^{19}$ S. Olibet, E. Vallat-Sauvain, and C. Ballif, Phys. Rev. B 76(3), 035326 (2007).

${ }^{20}$ C. Leendertz, N. Mingirulli, T. F. Schulze, J. P. Kleider, B. Rech, and L. Korte, Appl. Phys. Lett. 98(20), 202108 (2011).

${ }^{21}$ W. Favre, J. Coignus, N. Nguyen, R. Lachaume, R. Cabal, and D. Munoz, Appl. Phys. Lett. 102(18), 181118 (2013).

${ }^{22}$ D. Adachi, J. L. Hernández, and K. Yamamoto, Appl. Phys. Lett. 107(23), $233506(2015)$
${ }^{23}$ J. Kido, K. Hongawa, K. Okuyama, and K. Nagai, Appl. Phys. Lett. 64(7), 815 (1994).

${ }^{24}$ S. Lamansky, R. C. Kwong, M. Nugent, P. I. Djurovich, and M. E. Thompson, Org. Electron. 2(1), 53 (2001).

${ }^{25}$ B. Kippelen and J. L. Bredas, Energy Environ. Sci. 2(3), 251 (2009).

${ }^{26}$ G. Dennler, M. C. Scharber, and C. J. Brabec, Adv. Mater. 21(13), 1323 (2009).

${ }^{27}$ C. Deibel and V. Dyakonov, Rep. Prog. Phys. 73(9), 096401 (2010).

${ }^{28}$ E. Voroshazi, B. Verreet, A. Buri, R. Müller, D. Di Nuzzo, and P. Heremans, Org. Electron. 12(5), 736 (2011).

${ }^{29}$ C. Thompson and J. M. J. Fréchet, Angew. Chem., Int. Ed. 47(1), 58 (2008).

${ }^{30}$ J. Schmidt, V. Titova, and D. Zielke, Appl. Phys. Lett. 103(18), 183901 (2013).

${ }^{31}$ D. Zielke, A. Pazidis, F. Werner, and J. Schmidt, Sol. Energy Mater. Sol. Cells 131, 110 (2014).

${ }^{32}$ Y. Zhang, W. Cui, Y. Zhu, F. Zu, L. Liao, S. T. Lee, and B. Sun, Energy Environ. Sci. 8(1), 297 (2015).

${ }^{33}$ Y. Xu, J. Peng, J. Jiang, W. Xu, W. Yang, and Y. Cao, Appl. Phys. Lett. 87(19), 193502 (2005).

${ }^{34}$ X. Zhang, Z. Wu, D. Wang, D. Wang, R. He, and X. Hou, Appl. Surf. Sci. 256(14), 4468 (2010).

${ }^{35}$ A. Abate, T. Leijtens, S. Pathak, J. Teuscher, R. Avolio, M. E. Errico, J. Kirkpatrik, J. M. Ball, P. Docampo, I. McPherson, and H. J. Snaith, Phys. Chem. Chem. Phys. 15(7), 2572 (2013).

${ }^{36}$ J. H. Heo, S. H. Im, J. H. Noh, T. N. Mandal, C.-S. Lim, J. A. Chang, Y. H. Lee, H.-J. Kim, A. Sarkar, K. Nazeeruddin, M. Gratzel, and S. I. Seok, Nat. Photonics 7(6), 486 (2013).

${ }^{37}$ A. Descoeudres, L. Barraud, S. De Wolf, B. Strahm, D. Lachenal, C. Guerin, Z. C. Holman, F. Zicarelli, B. Demaurex, J. P. Seif, J. Holovsky, and C. Ballif, Appl. Phys. Lett. 99(12), 123506 (2011).

${ }^{38}$ A. Descoeudres, Z. C. Holman, L. Barraud, S. Morel, S. De Wolf, and C. Ballif, IEEE J. Photovoltaics 3(1), 83 (2013).

${ }^{39}$ S. De Wolf and G. Beaucarne, Appl. Phys. Lett. 88(2), 022104 (2006).

${ }^{40}$ Y. H. Kim, C. Sachse, M. L. Machala, C. May, L. Müller-Meskamp, and K. Leo, Adv. Funct. Mater. 21(6), 1076 (2011).

${ }^{41}$ Z. C. Holman, S. De Wolf, and C. Ballif, Light: Sci. Appl. 2(10), e106 (2013).

${ }^{42}$ Z. C. Holman, A. Descoeudres, S. De Wolf, and C. Ballif, IEEE J. Photovoltaics 3(4), 1243 (2013).

${ }^{43}$ A. Dabirian, S. Martin de Nicolas, B. Niesen, A. Hessler-Wyser, S. De Wolf, M. Morales-Masis, and C. Ballif, Adv. Mater. Interfaces 3(3), $1500462(2015)$ 